\title{
Meilleures publications 2011
}

\author{
(C) Springer-Verlag France 2011
}

\section{3}

\section{Hémorroïdes, fissures \\ J.D. Zeitoun}

Proctologie médico-interventionnelle, groupe hospitalier DiaconessesCroix-Saint-Simon, site Reuilly, 18, rue du Sergent-Bauchat, F-75012 Paris, France

Malgré les éclaircissements récents de la physiopathologie de la fissure anale, la question du traitement optimal reste très controversée. Aucune stratégie concernant les thérapeutiques pharmacologiques ne s'est imposée de façon incontestable. En cas d'échec de ces traitements médicaux, l'indication chirurgicale est consensuelle mais, là encore, il existe une grande dispersion des pratiques. Si la sphinctérotomie latérale interne (SLI) reste théoriquement le traitement de référence, la recherche d'un traitement chirurgical alternatif n'exposant pas aux troubles de la continence a conduit au développement et à l'évaluation d'autres techniques.

Une équipe britannique a publié les résultats d'une étude cas-témoins comparant la SLI à une technique de lambeau d'avancement cutané (LAC) chez 100 patients (50 patients dans chaque groupe) ayant une fissure anale chronique résistant à deux lignes de traitement médical [1]. Quatre-vingt-quatorze pour cent des patients avaient une douleur anale, $77 \%$ des saignements et seulement $15 \%$ étaient constipés. Les suivis moyens étaient respectivement de 20 et 22,5 mois dans le groupe LAC et SLI. La cicatrisation de la fissure était obtenue dans 96 et $88 \%$ des cas respectivement $(p=0,27)$. Une résolution des symptômes était rapportée chez 90 et $72 \%$ des patients respectivement $(p=0,04)$. Un taux plus élevé de réadmission était observé après SLI ( 8 vs $4 \%$ ), mais cette différence n'était pas significative $(p=0,35)$. Aucune incontinence fécale n'était à déplorer dans l'effectif global.

Les auteurs concluaient à la supériorité du LAC en termes de cicatrisation et de guérison des symptômes, et déduisaient de leurs résultats une moindre morbidité de leur technique. Malgré ces conclusions peut-être trop affirmatives, il semble peu probable que cet article impose le LAC comme une technique de référence. La méthodologie de l'étude l'explique en grande partie et on manque encore d'un, voire de plusieurs essai(s) randomisé(s) comparatif(s) entre SLI et les autres traitements chirurgicaux. Pour beaucoup d'auteurs, les techniques de lambeaux doivent être réservées aux patients n'ayant pas d'hypertonie sphinctérienne ou ayant des antécédents de chirurgie proctologique.

\section{Pathologie hémorroïdaire}

À l'instar de l'année dernière, le débat scientifique autour de la pathologie hémorroïdaire semble encore focalisé sur la compétition ouverte entre hémorroïdopexie de Longo et ligature sous contrôle doppler des artères hémorroïdales (désignée fréquemment par les abréviations HAL pour haemorrhoidal artery ligation ou THD pour transanal haemorrhoid dearterialization). Pourtant, une étape semble avoir été franchie puisque la précédente édition des Journées mettait en avant deux séries de patients opérés par HAL sans comparaison avec une autre technique, alors que cette année, il est possible d'analyser les résultats d'un essai contrôlé et randomisé multicentrique italien confrontant HAL et hémorroïdopexie de Longo [2]. L'objectif principal de l'étude était de comparer l'efficacité des deux techniques. Cent soixante-neuf patients ayant des hémorroïdes de grade III de Goligher ont été inclus et randomisés. La durée moyenne de suivi était de 17 mois. Le taux de complications postopératoires qualifiées de mineures était comparable entre les deux groupes (30,6 et 32,1\% respectivement pour les patients opérés par HAL et par Longo, $p=\mathrm{ns})$. La douleur postopératoire — spontanée ou à la défécation - était moins fréquemment observée dans le groupe HAL, mais la différence avec le groupe Longo n'était pas statistiquement significative. Les complications postopératoires tardives étaient statistiquement plus fréquentes après hémorroïdopexie par rapport au HAL $(p=0,028)$. Concernant l'efficacité, une différence était observée en faveur du Longo mais elle n'était pas statistiquement significative : persistance d'une pathologie hémorroïdaire chez respectivement 12 et 6 patients dans les groupes HAL et Longo $(p=0,14)$. Dix et six patients nécessitaient un nouveau traitement de leurs hémorroïdes dans les groupes respectifs HAL et Longo $(p=0,34)$. Aucune différence n'était observée entre les deux bras en termes d'incontinence postopératoire, mais une dyschésie était plus fréquente après Longo selon l'ODS $(p<0,02)$. Enfin, la durée de séjour était statistiquement plus longue après hémorroïdopexie par rapport au HAL : 1,31 vs 1,14 jour respectivement $(p=0,03)$. Les auteurs de cette étude concluaient à l'efficacité à moyen terme des deux techniques, avec un "plus » pour le HAL en raison de sa supériorité de ratio coût/efficacité, et sans différence en termes de douleur postopératoire et d'efficacité. D'autres études comparatives à la méthodologie solide sont attendues.

\section{Références}

1. Patel SD, Oxenham T, Praveen BV (2011) Medium-term results of anal advancement flap compared with lateral sphincterotomy for the treatment of anal fissure. Int J Colorectal Dis 26:1211-4 [Epub 2011 May 12]

2. Infantino A, Altomare DF, Bottini C, et al (2011) Prospective randomised multicenter study comparing Stapler Haemorrhoidopexy (SH) with Doppler guided Transanal Haemorrhoid Dearterialization (THD) for III degree haemorrhoids. Colorectal Dis doi:10.1111/j.1463-1318 [Epub ahead of print] 